\title{
Whole Genome Sequence of Sphingobacterium sp. G1-14, a Strain With Effective Paichongding Biodegradation
}

\author{
Peng Yin (Corresponding author) \\ Laboratory of Applied Microbiology, School of Pharmaceutical Engineering \& Life Science, \\ Changzhou University, Changzhou, China \\ Email: yinpengdevil@foxmail.com \\ Suyu Liu \\ Laboratory of Applied Microbiology, School of Pharmaceutical Engineering \& Life Science, \\ Changzhou University, Changzhou, China \\ Email: 490385680@qq.com \\ Xingke $\mathrm{Wu}$ \\ Laboratory of Applied Microbiology, School of Pharmaceutical Engineering \& Life Science, \\ Changzhou University, Changzhou, China \\ Email: 1092678638@qq.com
}

Received: December 6, 2018

Accepted: December 24, 2018

doi:10.5296/jbls.v10i1.14000

URL: https://doi.org/10.5296/jbls.v10i1.14000

\begin{abstract}
In this study, we obtained a Paichongding (RR/SS-IPP) degrading Sphingobacterium sp. G1-14 by UV irradiation of the original strain G1-13. This new mutant strain showed excellent RR/SS-IPP degradation performance, and the degradation of ratio was up to 30 per cent after 7 days. Subsequently, we determined the mutant strain G 1-14 as Sphingobacterium based on the phylogenomic analyses. The circular chromosome of Sphingobacterium sp. G1-14 was presented in this paper by Illumina Hiseq platform combined with a third-generation sequencing platform. 5583 protein-coding sequences of the complete genome sequence were obtained, which is beneficial to deduced genes related to RR/SS-IPP degradation.
\end{abstract}

Keywords: Paichongding, Sphingobacterium, Biodegradation, Biodegradation pathway, Whole genome 


\section{Introduction}

Insecticide plays an increasingly important role in modern agriculture for its highly resistance to farmland pests. In the past two or three decades, neonicotinoid insecticides have become one of the largest sales insecticides in more than 120 countries around the world. As a representative of insecticide, Paichongding (IPP,

1-((6-chloropydidin-3-yl)methyl)-7-methyl-8-nitro-5propoxy-1,2,3,5,6,7-hexahydroimidazo[ 1,2-a-]-pyridine), was developed by Jiangsu Kwin Co., Ltd. and East China University of Science and Technology in 2008 and showed excellent activity for anti-imidacloprid insects(Cai et al., 2015; Qin et al., 2012; H. Y. Wang et al., 2013). IPP is considered as the succedaneum of imidacloprid which has negative influence on wild bumble bee populations (Fu, Zhang, et al., 2013; Whitehorn, O'Connor, Wackers, \& Goulson, 2012). In China, 10\% IPP suspensions have been sprayed in farmland for pest prevention such as alfalfa aphids and brown planthopper since 2009(Fu, Wang, et al., 2013). IPP is expected to reach 1,000 tons in few years, which may pose a significant threat to soil and water (Cai et al., 2015). Many studies have demonstrated that microorganisms play vital roles in pesticide degradation (MAE, MARIT, AUSMES, KOIV, \& HEINAR, 1993; Vaitekunas, Gasparaviciute, Rutkiene, Tauraite, \& Meskys, 2016). Remarkably, Cai et al. has reported that the IPP could be biodegraded by Sphingobacterium sp. P1-3(Cai et al., 2015).

At present, genes and enzymes related to the degradation of RR/SS-IPP have not been reported. Based on the newly selected RR/SS-IPP efficient degrading bacteria, this study provides insights into the degradation mechanism of RR/SS-IPP from a genetic perspective through the whole-genome sequencing and bioinformatics analysis combined with liquid chromatography tandem high-resolution mass spectrometry (LC-MS/MS).

\section{Material and Methods}

\subsection{Chemicals and Microorganism Strain}

Paichongding, (IPP, chemical purity 98.3\%; Formula Weight, FW 366. Fig. 1) was obtained from Jiangsu Kesheng Company Ltd., HPLC grade methanol and acetonitrile were purchased from Burdick \& Jackson (MI, USA). All other reagents and common chemicals were analytical grade and purchased from Sinopharm Chemical Reagent Company (Shanghai, China).

Sphingobacterium sp. G1-14 (GenBank accession numbers: KP657689, CGMCC No. 10454) was irradiated from the original strain G1-13, which was isolated from soils in the south of Changzhou, Jiangsu province, China. It had good IPP-degrading activity and could use IPP as a sole carbon and energy source. Sphingobacterium sp. G1-14 was cultivated aerobically at $30{ }^{\circ} \mathrm{C}$ on Luria-Bertani (LB) broth. Stock cultures were stored in $20 \%$ glycerol at $-80{ }^{\circ} \mathrm{C}$.

\subsection{Biodegradation Assays}

To illustrate the biodegradation pathway of IPP in aquatic system, $250 \mathrm{~mL}$ glass flasks were used in all the degradation study and $150 \quad 1$ of IPP stock solution $\left(20 \mathrm{~g} \mathrm{l}^{-1}\right)$ was added into $100 \mathrm{ml}$ of BMM solution to give a final concentration of $30 \mathrm{mg} \mathrm{l}^{-1}$. And all the tests are three 


\section{Macrothink}

replicates. After culture for $72 \mathrm{~h}$, cells of Sphingobacterium sp. G1-14 were harvested by centrifugation $\left(21,000 \times g, 4{ }^{\circ} \mathrm{C}, 10 \mathrm{~min}\right)$ and washed twice with $0.85 \%(\mathrm{w} / \mathrm{w})$ sodium chloride solution.

\subsection{Extraction and Pretreatment}

The cultivation was collected by centrifugation $\left(21,000 \times g, 4{ }^{\circ} \mathrm{C}, 10 \mathrm{~min}\right)$. The supernatant was extracted with dichloromethane three times. The recovery of IPP ranged from $91.22 \%$ to 107.29, the relative standard deviation (RSD) was less than $7.58 \%$. The results showed that the extraction method is feasible and satisfactory for the analysis of IPP residues. The dichloromethane extracts were combined and concentrated through vacuum rotary evaporator at $40{ }^{\circ} \mathrm{C}$ to almost dry and then the extracts were dissolved in $1 \mathrm{ml}$ of methanol. IPP and its biodegradation intermediates were monitored and analyzed.

\subsection{Genomic DNA Extract and Sequence}

The commercial kits (PureLink ${ }^{\mathrm{TM}}$ Microbiome DNA Purification Kit, ThermoFisher Scientific) were used for extracting and purifying of genomic DNA from Sphingobacterium sp. G1-14. The genomic DNA was sequenced using Illumina Hiseq 2500 at BGI company (Shenzhen, China).

\section{Results}

As a chiral insecticide, IPP can be divided into four stereoisomers according to two asymmetrically substituted C atoms (Fig. 1). Compared with enantiomers RS-IPP and SR-IPP, enantiomers RR-IPP and SS-IPP exhibit higher stablility and difficulty in degradation ( $\mathrm{Fu}$, Wang, et al., 2013; J. Wang et al., 2016). In this study, we found a gram-negative mutant strain G1-14 from G1-13 through UV mutagenesis. As is shown in Fig 2, G1-14 in culture plates has higher growth rate and larger single colonies than G1-13. After seven days of fermentation, the IPP degradation rate was about 30\%, which was superior to original strain significantly. The phylogenetic tree based on 16S rRNA gene sequence was constructed using MEGA (v5.1) (Fig. 3). It could reveal the evolutionary status of Sphingobacterium sp. G1-14. 


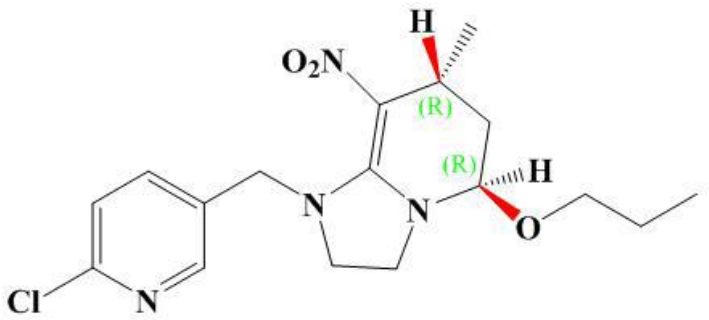

(5R,7R)-paichongding(RR-IPP)<smiles>CCCO[C@H]1C[C@@H](C)C([N+](=O)[O-])=C2N(Cc3ccc(Cl)nc3)CCN21</smiles>

(5S,7R)-paichongding(SR-IPP)

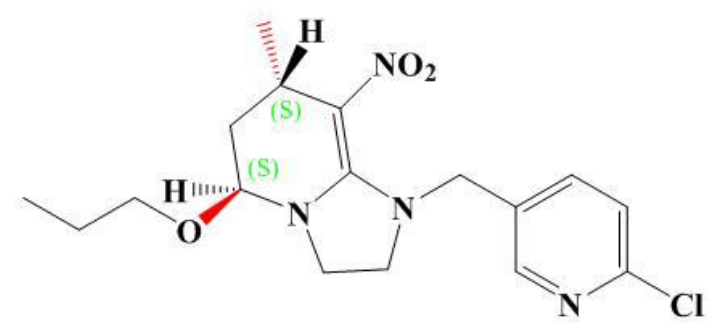

(5S,7S)-paichongding(SS-IPP)<smiles>CCCO[C@H]1C[C@H](C)C([N+](=O)[O-])=C2N(Cc3ccc(Cl)nc3)CCN21</smiles>

(5R,7S)-paichongding(RS-IPP)

Figure 1. Four stereoisomers structures of Paichongding (IPP): (A) 5R,7R-IPP (RR-IPP), (B) 5S,7S-IPP (SS-IPP), (C) 5S,7R-IPP (SR-IPP), and (D) 5R,7S-IPP (RS-IPP) and the chiral centers have been marked $\mathrm{R}$ or $\mathrm{S}$
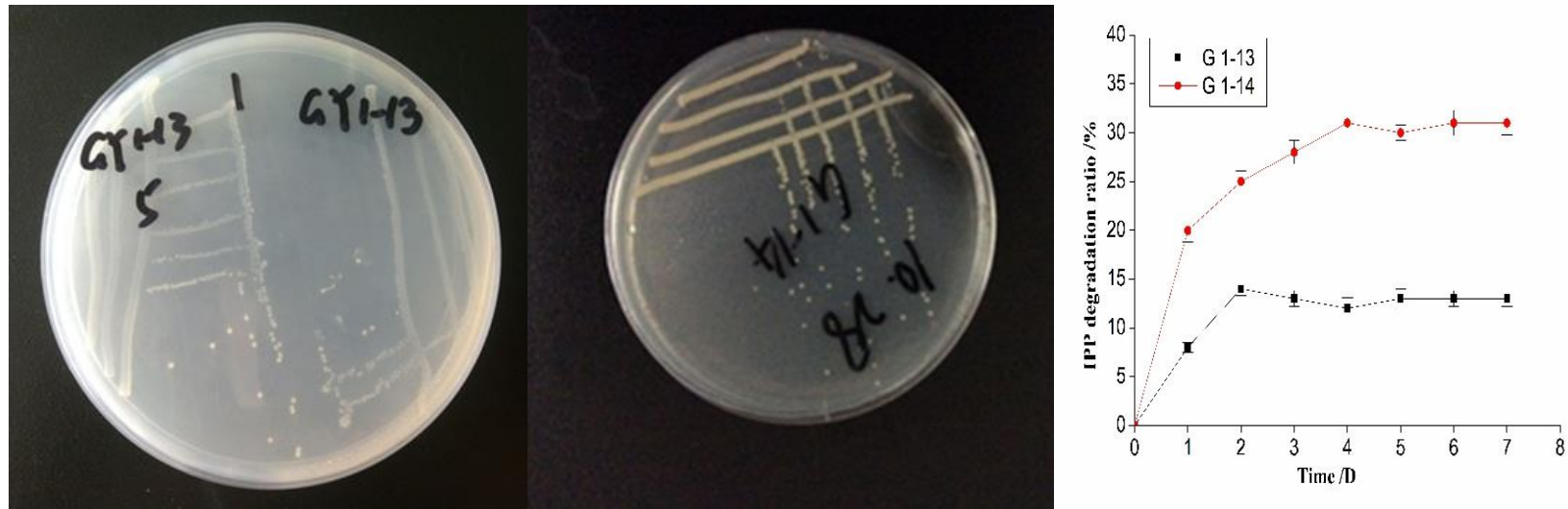

Figure 2. Basal medium plates colonies of strains G1-13 / G1-14 and the IPP degradation ratio by strains G1-13 / G1-14 within seven days 


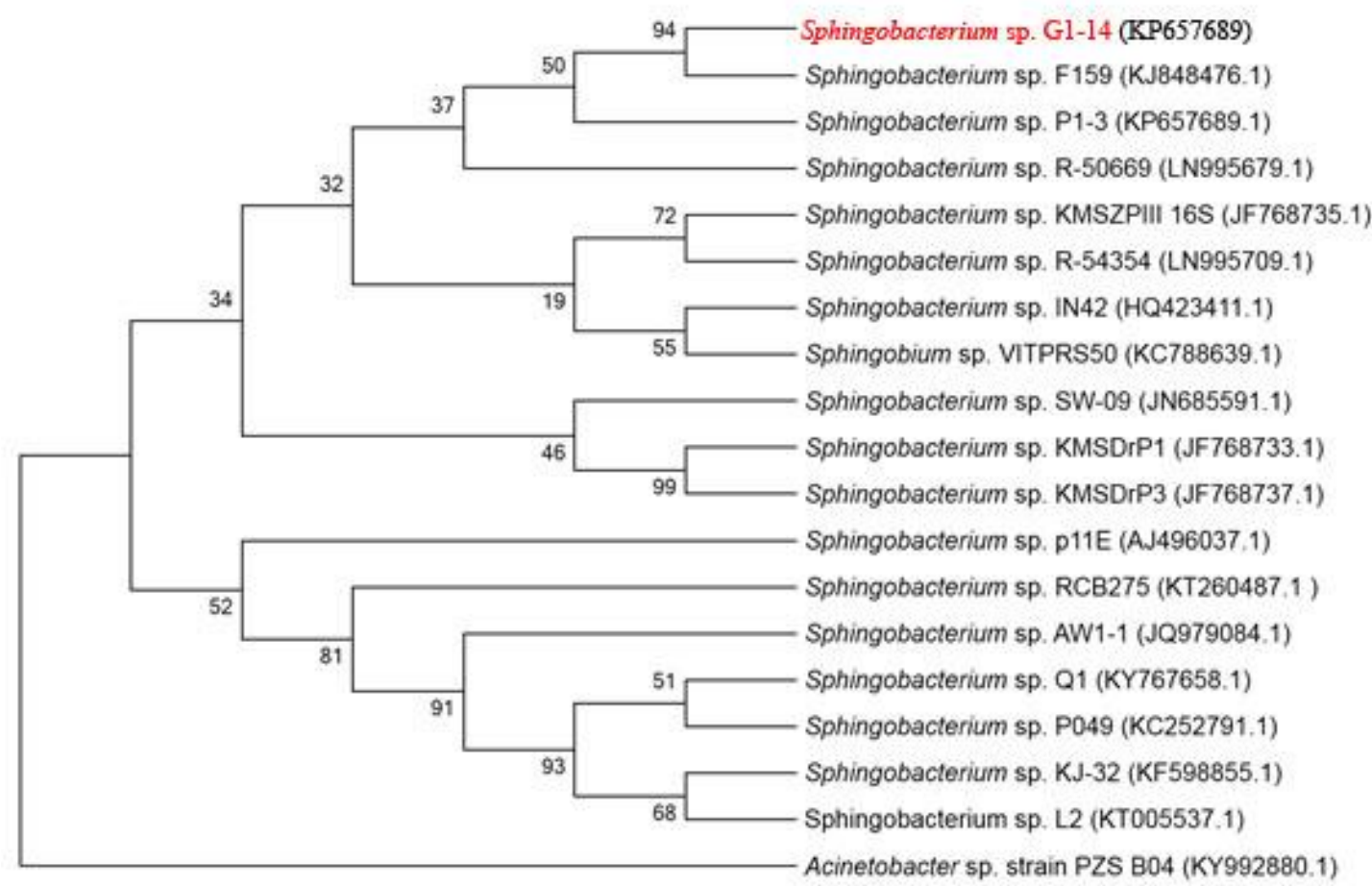

Figure 3. The phylogenetic tree was constructed based on 16S rRNA gene sequences showing the relationship between Sphingobacterium sp. G1-14 and other Sphingobacterium species.

The neighbor-joining method would help to inferred the evolutionary history of Sphingobacterium sp. G1-14

Our group attempted to speculate the conceivable metabolic pathway of RR/SS-IPP by Sphingobacterium sp. G1-14 though the liquid chromatography tandem high-resolution mass spectrometry (LC-MS/MS). Owing to the complicated mechanism of RR/SS-IPP, the whole genome sequence was added to provide insights about the degradation mechanism of RR/SS-IPP from the genetic point of view. The Sphingobacterium sp. G1-14 was incubated at $30^{\circ} \mathrm{C}, 180$ revolutions $\mathrm{min}^{-1}$ in $100 \mathrm{~mL}$ of $\mathrm{LB}$ medium. The genomic DNA was extracted using the Takara DNA kit and DNA concentration was measured using Qubit3.0 to ensure DNA quality for the subsequent experiments. Illumina HiSeq 2500 system combined with Pacfic Biosciences SMRT (PacBio RSII) have been used to complete genome sequence. The Illumina PE library and PacBio library generated $2021 \mathrm{Mb}$ data $(14,532,302$ reads with 335 bp average coverage) and $625 \mathrm{Mb}$ data (83839 subreads with 7,453 bp average length) respectively. We assembled the genome into one contig based on the reads obtained from Illumina PE library and PacBio library by SOAPdenovo (v2.04) and Celera Assembler (v8.0). GapCloser (v1.12) was used to complete the gap closure and confirm a 6,325,678 bp chromosome with circular topology(Koren et al., 2012). The function annotations of protein-coding genes (predicted by Glimmer 3.0) were obtained from BLASTP with COG, KEGG and Swiss-Prot databases (Delcher, Bratke, Powers, \& Salzberg, 2007). Finally, we indentified the rRNA and tRNA contained in the genome using RNAmmer (v1.2) and tRNAscan-SE (v1.3.1). The assembly result of Sphingobacterium sp. G1-14 genome revealed 


\section{I Macrothink}

Journal of Biology and Life Science ISSN 2157-6076 2019, Vol. 10, No. 1

that the circular chromosome contained $6,325,678$ bp with $\mathrm{G}+\mathrm{C}$ content of $39.83 \% .5583$ CDSs, 21 rRNA and 85 tRNA were identified in the chromosome respectively. The summary genome features of Sphingobacterium sp. G1-14 were summarized in Table 1 and Fig 4.

Table 1. Sphingobacterium sp. G1-14 genome features

\begin{tabular}{cc}
\hline Features & G1-14 \\
\hline Genome size (bp) & $6,325,678$ \\
GC content (\%) & 39.83 \\
Numbers of CDSs & 5583 \\
Numbers of rRNAs & 21 \\
Numbers of tRNAs & 85 \\
GeneBank accession & CP021381 \\
\hline
\end{tabular}

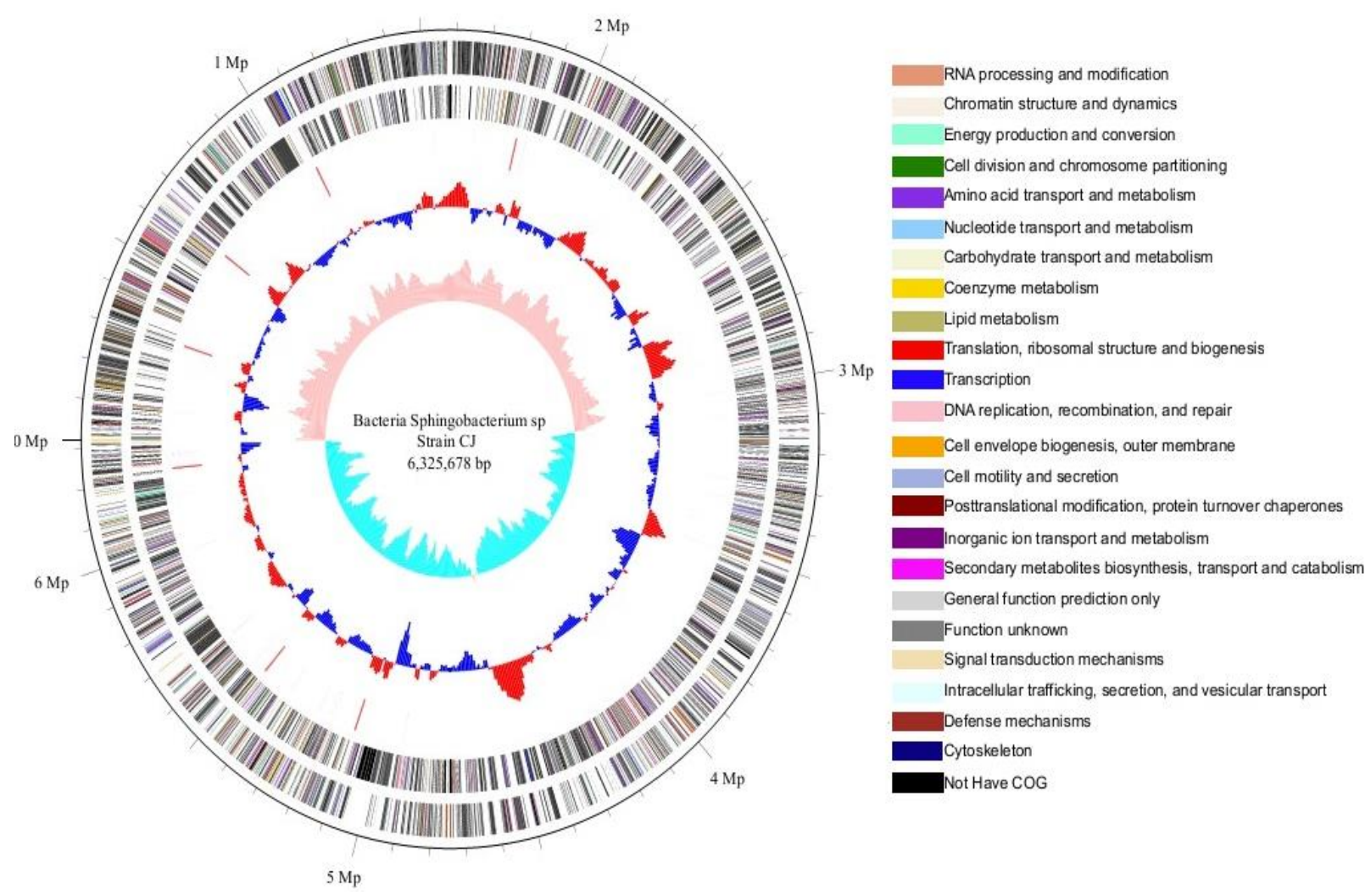

Figure 4. Genomic circle map of Sphingobacterium sp. G1-14. From the outside circle to the center, circle (1) shows the genomic size and each scale is $0.5 \mathrm{Mb}$. Circle (2) and circle (3) represent the predicted CDSs on negative for the positive chain and negative chain, colors according to COG functional categories. Circle (4) indicates the rRNA and tRNA genes.

Circle (5) shows the GC content 


\section{Macrothink}

\section{Discussion}

In combination with deduced metabolic pathways (J. Wang et al., 2016), we found that: (i) IPP and imidacloprid are structurally consistent in Phase I (Fig 5). KEGG pathway analysis suggested that CYP450 enzymes were not exist in the genome sequence. CYP450s were the only proven enzymes that involved in imidazole ring hydroxylation in imidacloprid with stable chloropyridine ring (Casida, 2011; Fu, Zhang, et al., 2013; Schulz-Jander, Leimkuehler, \& Casida, 2002) . Our results deduced that the hydroxylation of IPP occurs in the tetrahydropyridine ring based on six hydroxylases in the genome. (ii) According to the KEGG database, MqnB was found in the chromosome of G1-14 which could hydrolyze the C-N bond attached to the imidazole (Hiratsuka et al., 2008). The formation of I1 and I2 were obtained from the removal of $\mathrm{C}-\mathrm{N}$ bonds between 2-chloro-5-ethylpyridine and 8-amino octahydroimidazo [1,2-alpha] -pyridin-7-ol. Based on these observations, we hypothesized that the break of this $\mathrm{C}-\mathrm{N}$ bond was mainly due to the candidate enzyme MqnB.

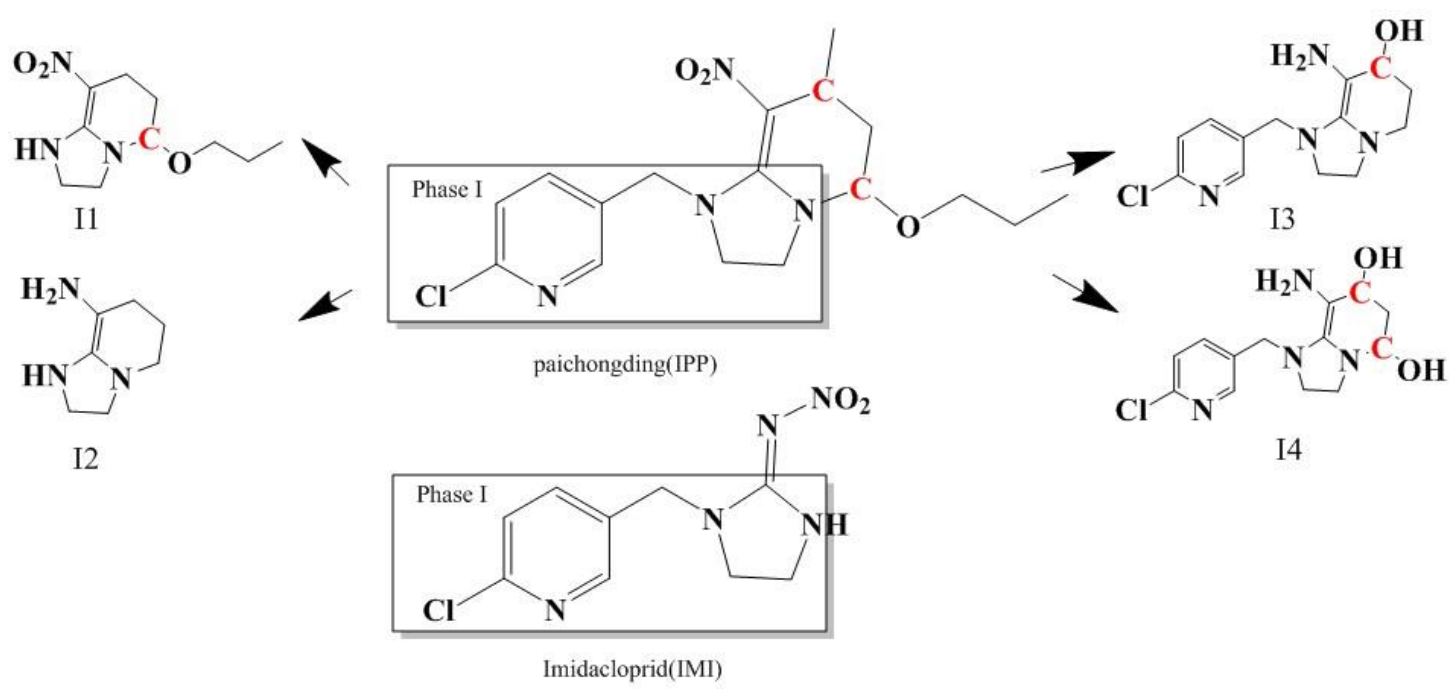

Figure 5. Paichongding (IPP) and Imidacloprid (IMI) Chemical Structural Formulas and RR/SS-IPP metabolites (I1, I2, I3 and I4) by Sphingobacterium sp. G1-14.

\section{Conclusion}

In this study, we obtained the whole-genome sequence of the Sphingobacterium sp. G1-14. Through bioinformatics analysis, hydroxylation of RR/SS-IPP may occur on relatively active tetrahydropyridine rings. From the metabolic point of view, the candidate enzyme MqnB might be responsible for the removal of the chloropyridine ring.

\section{Competing interests}

The authors declare that they have no competing interests.

\section{Funding}

The research was financially funded by grants from the National Natural Science Foundation of China (NSFC) (Project 11275033) and Natural Science Foundation of Jiangsu Province (BK20151185). 


\section{Authors' contributions}

PY, SL, WX and JG carried out the experiments; PY and XZ wrote the paper; XZ and ZC designed the research; ZC obtained the funding. All authors read and approved the final manuscript.

\section{References}

Cai, Z. Q., Zhang, W. J., Li, S. S., Ma, J. T., Wang, J., \& Zhao, X. Y. (2015). Microbial Degradation Mechanism and Pathway of the Novel Insecticide Paichongding by a Newly Isolated Sphingobacterium sp P1-3 from Soil. Journal of Agricultural and Food Chemistry, 63(15), 3823-3829. https://doi.org/10.1021/acs.jafc.5b00706

Casida, J. E. (2011). Neonicotinoid Metabolism: Compounds, Substituents, Pathways, Enzymes, Organisms, and Relevance. Journal of Agricultural and Food Chemistry, 59(7), 2923-2931. doi:10.1021/jf102438c

Delcher, A. L., Bratke, K. A., Powers, E. C., \& Salzberg, S. L. (2007). Identifying bacterial genes and endosymbiont DNA with Glimmer. Bioinformatics, 23(6), 673-679. https://doi.org/10.1093/bioinformatics/btm009

Fu, Q. G., Wang, Y. C., Zhang, J. B., Zhang, H. X., Bai, C., Li, J. Y., ... Li, Z. (2013). Soil Microbial Effects on the Stereoselective Mineralization, Extractable Residue, Bound Residue, and Metabolism of a Novel Chiral Cis Neonicotinoid, Paichongding. Journal of Agricultural and Food Chemistry, 61(32), 7689-7695. https://doi.org/10.1021/jf4015153

Fu, Q. G., Zhang, J. B., Xu, X. Y., Wang, H. Y., Wang, W., Ye, Q. F., \& Li, Z. (2013). Diastereoselective Metabolism of a Novel Cis-Nitromethylene Neonicotinoid Paichongding in Aerobic Soils. Environmental Science \& Technology, 47(18), 10389-10396. https://doi.org/10.1021/es4023738

Hiratsuka, T., Furihata, K., Ishikawa, J., Yamashita, H., Itoh, N., Seto, H., \& Dairi, T. (2008). An alternative menaquinone biosynthetic pathway operating in microorganisms. Science, 321(5896), 1670-1673. https://doi.org/10.1126/science.1160446

Koren, S., Schatz, M. C., Walenz, B. P., Martin, J., Howard, J. T., Ganapathy, G., . . Phillippy, A. M. (2012). Hybrid error correction and de novo assembly of single-molecule sequencing reads. Nature Biotechnology, 30(7), 692-+. https://doi.org/10.1038/nbt.2280

MAE, A. A., MARIT, R. O., AUSMES, N. R., KOIV, V. M., \& HEINAR, A. L. (1993). Characterization of a new 2,4-dichlorophenoxyacetic acid degrading plasmid pEST4011: physical map and localization of catabolic genes. Journal of General Microbiology(139), 3 165-163 170.

Qin, X. W., Chen, Y., Zhang, J., Liu, J., Liu, Q., Yuan, F. H., \& Zhang, R. J. (2012). Toxic Effects of Paichongding on Brown Planthopper, Nilaparvata lugens (Stal) (Homoptera: Delphacidae). Journal of Entomological Science, 47(4), 297-308.

Schulz-Jander, D. A., Leimkuehler, W. M., \& Casida, J. E. (2002). Neonicotinoid insecticides: 


\section{Macrothink}

Reduction and cleavage of imidacloprid nitroimine substituent by liver microsomal and cytosolic enzymes. Chemical Research in Toxicology, 15(9), 1158-1165. https://doi.org/10.1021/tx0200360

Vaitekunas, J., Gasparaviciute, R., Rutkiene, R., Tauraite, D., \& Meskys, R. (2016). A 2-Hydroxypyridine Catabolism Pathway in Rhodococcus rhodochrous Strain PY11. Appl Environ Microbiol, 82(4), 1264-1273. https://doi.org/10.1128/AEM.02975-15

Wang, H. Y., Yang, Z., Liu, R. Y., Fu, Q. G., Zhang, S. F., Cai, Z. Q., . . . Li, Z. (2013). Stereoselective uptake and distribution of the chiral neonicotinoid insecticide, Paichongding, in Chinese pak choi (Brassica campestris ssp chinenesis). Journal of Hazardous Materials, 262, 862-869. https://doi.org/10.1016/j.jhazmat.2013.09.054

Wang, J., Chen, J., Zhu, W. J., Ma, J. T., Rong, Y., \& Cai, Z. G. (2016). Isolation of the Novel Chiral Insecticide Paichongding (IPP) Degrading Strains and Biodegradation Pathways of RR/SS-IPP and SR/RS-IPP in an Aqueous System. Journal of Agricultural and Food Chemistry, 64(40), 7431-7437. https://doi.org/10.1021/acs.jafc.6b02862

Whitehorn, P. R., O'Connor, S., Wackers, F. L., \& Goulson, D. (2012). Neonicotinoid Pesticide Reduces Bumble Bee Colony Growth and Queen Production. Science, 336(6079), 351-352. https://doi.org/10.1126/science.1215025

\section{Copyright Disclaimer}

Copyright for this article is retained by the author(s), with first publication rights granted to the journal.

This is an open-access article distributed under the terms and conditions of the Creative Commons Attribution license (http://creativecommons.org/licenses/by/3.0/). 